\title{
Is Condensation-Induced Atmospheric Dynamics a New Theory of the Origin of the Winds?
}

\author{
A. JARAMiLlo AND O. J. MESA \\ Facultad de Minas, Departamento de Geociencias y Medio Ambiente, Universidad Nacional de Colombia, Medellin, Colombia \\ D. J. RAYMOND \\ Physics Department, New Mexico Institute of Mining and Technology, Socorro, New Mexico
}

(Manuscript received 3 October 2017, in final form 28 June 2018)

\begin{abstract}
The hypothesis of "condensation-induced atmospheric dynamics" proposes that a previously unstudied force associated with condensation is the driver of atmospheric motions, explaining phenomena like cyclones, monsoon circulations, and even the Hadley circulation. This hypothesis caused significant interest in the academic community, but it also produced substantial controversy, receiving numerous criticisms from experts who have serious doubts about the existence or importance of this force. In this paper, we show that the alleged new force is based on an unbalanced internal force within the atmospheric gas. Therefore, the dynamic effects attributed to this force are not physically possible, pointing to the violation of Newton's third law. We also reiterate that the role of the water cycle in the standard theory is essential to explain major atmospheric circulations but without physical inconsistencies.
\end{abstract}

\section{Introduction}

Makarieva and Gorshkov (2007) proposed a new hypothesis where a "previously unstudied force" associated with condensation is the driver of low-level circulations, explaining phenomena like cyclones, monsoon circulations, and even the Hadley circulation (e.g., Makarieva and Gorshkov 2007, 2009a,b,c, 2010; Gorshkov et al. 2012; Makarieva et al. 2013, 2014, 2015, 2017). This hypothesis, called by its authors condensation-induced atmospheric dynamics [the Makarieva-Gorshkov hypothesis $(\mathrm{MGH})$ ], describes an atmospheric process where this new force drives horizontal fluxes of moist air from areas of weak to strong evaporation, enhancing precipitation where evaporation is highest. MGH proposes the idea that forests use this principle to suck in moist air from the oceans into the continents, maintaining the hydrological cycle inland and providing a physical mechanism to explain the relationship between forests and climate.

MGH generated interest in the academic community, but it also received considerable criticism from experts who have serious doubts about the existence or importance of this force. An unusual editorial comment at the

Corresponding author: A. Jaramillo, ajarami2@unal.edu.co end of Makarieva et al. (2013) clearly states the controversy: "The paper is highly controversial, proposing a fundamentally new view that seems to be in contradiction to common textbook knowledge. [...] The majority of reviewers and experts in the field seem to disagree, whereas some colleagues provide support, and the handling editor (and the executive committee) are not convinced that the new view presented in the controversial paper is wrong."

Despite the negative criticism by experts suggesting that there is something wrong with the physics of $\mathrm{MGH}$, it seems that the only published criticism of this hypothesis is the work of Meesters et al. (2009), which analyzes the physical impossibility of the new force proposed by Makarieva and Gorshkov (2007). Nevertheless, Makarieva and Gorshkov (2009c) suggest that Meesters et al. (2009) misunderstood the mechanism raised by MGH. Later publications, like Makarieva et al. (2013), increased the controversy about these ideas. Consequently, there is a need for a strong physical argument resolving the questions concerning $\mathrm{MGH}$.

We will demonstrate from mechanical and thermodynamical analyses that the alleged new force is based on an unbalanced internal force within the atmospheric gas violating Newton's third law. Therefore, the dynamic 
effects attributed to this force are not physically possible, pointing to deep problems in the physics of the proposal. To explain the deficiencies in the physics of MGH, we will restrict our arguments to a simplified view of moist convection, following an approach similar to that in standard textbooks in atmospheric thermodynamics, but our approach will be rigorous.

This paper is organized as follows. Section 2 presents a brief review of the ideas that lead to the appearance of the force proposed by MGH. Section 3 explains from a mechanical and thermodynamical perspective that the force proposed by MGH violates Newton's third law. In section 4 , we present a brief view of the role of the hydrologic cycle in the atmospheric circulation. Finally, section 5 presents the main conclusions of this work.

\section{The evaporative force}

MGH proposes that the main driver of low-level circulations is a new and overlooked "evaporative force" $f_{e}$, also called the condensation force or the evaporativecondensational force. To explain this force, MGH focuses on the effects of adiabatic condensation on the hydrostatic balance of the atmosphere.

For large-scale motions, the atmosphere is to a high degree of accuracy in hydrostatic balance (Holton and Hakim 2013, p. 45), that is, the gravity force is balanced by the vertical component of the pressure gradient force,

$$
-\frac{\partial p}{\partial z}=\frac{p}{h}
$$

where $h=R T / M g$ is the scale height for the mixture, $R$ is the universal gas constant, $T$ is the absolute temperature, $M$ is the molar mass of the mixture (Wallace and Hobbs 2006, p. 69), and $g$ is the gravity acceleration constant.

In the absence of condensation and evaporation, all components of the atmosphere, including the water vapor, would be well mixed and all would exhibit the above scale height $h$; therefore

$$
-\frac{\partial p_{i}}{\partial z}=\frac{p_{i}}{h}
$$

where $p_{i}$ is the partial pressure of the $i$ th component.

Note that this is different from component hydrostatic equilibrium (called "aerostatic balance" in MGH), where the partial pressures of the individual gases decrease with a scale height inversely proportional to the molecular weight of each gas $M_{i}$ in the absence of molecular collisions between components (i.e., each gas behaves as if it alone occupied the entire volume of the original mixture),

$$
-\frac{\partial p_{i}}{\partial z}=\frac{p_{i}}{h_{i}}
$$

where $h_{i}=R T / M_{i} g$. In the absence of turbulent mixing, the whole atmosphere could be in hydrostatic equilibrium with each component in aerostatic equilibrium, though the total pressure and density could not be represented by a single exponentially decaying curve with height in this case. We group all of the dry components together with a common scale height $h_{d}$ and denote the aerostatic scale height of the water vapor as $h_{v}$. Obviously, (2) and (3) cannot be both true; the analysis of which of the two is relevant for prevailing atmospheric conditions and what are its implications is at the center of this paper.

MGH asserts that a steep decline of temperature with height in a saturated column prevents the water vapor from fulfilling (3) because of condensation and fallout of precipitation, creating a force $f_{e}$ that disturbs the hydrostatic balance of the air as a whole. Based on the example of the pressure profiles in moist columns presented by Makarieva et al. (2013, their section 3), we can explain the reasoning that MGH uses to justify the existence of $f_{e}$. We can split their argument into four steps: 1) They start with a static isothermal moist column; the column obeys hydrostatic balance and all their components are in aerostatic equilibrium (i.e., not well mixed), including water vapor, which is saturated at the surface only. 2) They introduce a moist adiabatic lapse rate of temperature in the static column. 3) Now, in this case, the Clausius-Clapeyron relationship dictates that the air at each level is too cold to hold the amount of water vapor that maintains aerostatic balance. Water vapor in excess condenses and falls out, saturating the column at each level and resulting in a compressed vertical profile of water vapor compared to the profile needed for aerostatic equilibrium. 4) $\mathrm{MGH}$ asserts that with the onset of condensation, the water vapor is the only component that is strongly out of aerostatic balance as a result of the large vertical gradient of vapor pressure dictated by the Clausius-Clapeyron equation. This equation may be written in a form similar to the hydrostatic equation (Makarieva and Gorshkov 2007),

$$
-\frac{\partial p_{v}^{*}}{\partial z}=\frac{p_{v}^{*}}{h_{c}},
$$

where $p_{v}^{*}$ is the saturated water vapor pressure, and

$$
h_{c}=\frac{R_{v} T^{2}}{L_{L}\left(-\frac{\partial T}{\partial z}\right)},
$$

with $R_{v}=R / M_{v}$ being the gas constant for water vapor and $L_{L}$ being the latent heat of condensation. This means that the vertical gradient for the water vapor will 
be dictated by a scale height $h_{c}$, different from $h_{v}$ (e.g., Makarieva and Gorshkov 2007; Makarieva et al. 2013; Gorshkov et al. 2012). Therefore $-\partial p_{v} / \partial z \neq p_{v} / h_{v}$, and there appears a force $f_{e}$ on the water vapor component that can be written as

$$
f_{e}=-\frac{\partial p_{v}}{\partial z}-\frac{p_{v}}{h_{v}}
$$

In the presence of this force, the hydrostatic balance of the air as a whole is disrupted and its readjustment is at the expense of horizontal motion toward the condensation area producing the air fluxes that MGH describes as the best explanation for the origin of the winds.

Later, Gorshkov et al. (2012) and Makarieva et al. (2013) gave a conflicting definition for $f_{e}$ :

$$
f_{e}=-\frac{\partial p_{v}}{\partial z}-\frac{p_{v}}{h}
$$

that is, (7) uses $h$ instead of $h_{v}$ in (6). The authors do not explicitly explain the change in the definition of this force, but in other words, they are saying that condensation prevents the water vapor from fulfilling (2) instead of (3). In the next section, we explain the main problem with both definitions (6) and (7).

Another point worth noting is that MGH is distinctly different from the mass sink problem, where the mass continuity equation for the atmosphere has a sourcesink term due to the addition of water vapor by surface evaporation and its subtraction due to precipitation formation. This is a well-known effect (Raymond 2013) but is generally small, which is why many models do not include it. However, there may be some special cases with heavy rainfall where it might be marginally significant (e.g., Lackmann and Yablonsky 2004) or of firstorder importance in hypothetical atmospheres with elevated water vapor mixing ratios. The force proposed by MGH is a modification to the vertical momentum budget, not the mass budget, and so is distinct. Here, we restrict ourselves to the dynamical aspects of $\mathrm{MGH}$, and a discussion of the mass continuity equation in $\mathrm{MGH}$ will be postponed for a future paper.

\section{The evaporative force and Newton's third law}

The key to understanding the problem with MGH's proposed force is by identifying that this hypothesis, in general, does not address the role of dry air correctly in the mixture, in particular during the process of condensation. In the real world, the vertical expansion of the water vapor column due to the difference between its actual and aerostatic scale heights is frustrated by the dry atmosphere. Water vapor molecules slowly diffuse through the dry component, a process that is manifested on microscopic scales by collisions between water vapor and dry-air molecules. Although it is true that only water vapor is undergoing phase changes, this process occurs immersed inside a matrix of dry air that reacts to the changes during condensation. Therefore, the mixture of moist air must be treated carefully to avoid neglecting these essential interactions as MGH does. Here we will approach the problem from a mechanical point of view as well as from a thermodynamic point of view, where the effects of condensation will be more transparent.

To study the vertical motion of a parcel of moist air, one must apply Newton's second law. For a mixture of dry air (with all components considered together) and water vapor, the vertical component of total force per unit volume is the sum of the forces on each component,

$$
\begin{aligned}
F_{z} & =\left(-\frac{\partial p_{d}}{\partial z}-g \rho_{d}+F_{v d}\right)+\left(-\frac{\partial p_{v}}{\partial z}-g \rho_{v}+F_{d v}\right) \\
& =-\frac{\partial p}{\partial z}-g \rho+F_{v d}+F_{d v}=\rho a_{z},
\end{aligned}
$$

where $p_{d}$ and $p_{v}$ are the partial pressures of dry air and vapor; $\rho_{d}$ and $\rho_{v}$ are the corresponding densities $\left(\rho=\rho_{d}+\rho_{v}\right.$ and $\left.p=p_{d}+p_{v}\right) ; a_{z}$ is the vertical acceleration of the air; and $g$ is the gravitational acceleration. For simplicity, we ignored the frictional force that the environment does on the parcel.

The quantities $F_{v d}$ and $F_{d v}$ are, respectively, the force of the vapor on the dry air and the force of the dry air on the vapor, as mediated by molecular collisions between the two components. These are internal forces, since they act between components of the total system, which is the mixture of dry air and vapor. Newton's third law states that

$$
F_{v d}=-F_{d v}
$$

This relationship is unequivocal. It does not depend on any other properties of the system, such as its acceleration. (See any elementary physics text for further discussion.)

The "evaporative force" of MGH, as expressed in (6), is nothing more than the force of the water vapor on the dry component of the atmosphere $F_{v d}$. As $-\partial p_{v} / \partial z>g \rho_{v}$, as stressed by MGH, this force is upward. If the air parcel is not undergoing vertical acceleration, then $F_{v d}=f_{e}$, as defined by (6). For weak vertical accelerations, this relation is still approximately true.

The flaw in MGH's theory is now clear: MGH includes $F_{v d}$ in the vertical motion equation while omitting $F_{d v}$. This is a clear violation of Newton's third law; both of these forces must be either included or excluded. It does not matter which, since they cancel out if included. 
The version of MGH's theory expressed in (7) is also incorrect as it postulates a somewhat modified unbalanced internal force, albeit with even less plausible reasoning. The entire basis for MGH's theory of a "previously unstudied force" thus collapses because of an elementary error in their understanding of fundamental physics. $\mathrm{Cu}$ riously, Meesters et al. (2009) came very close to making a decisive argument against MGH. However, they failed to point out the crucial role of Newton's third law in disproving this hypothesis, allowing dubious arguments by Makarieva and Gorshkov (2009c) to muddy the waters.

It is important to point out here the role of condensation in this picture. From a thermodynamic point of view, we can understand how condensation can affect (8) by studying the thermodynamic changes of a moist saturated parcel that is undergoing condensation. We can explore this with the help of the specific moist entropy equation, which links the dry partial pressure $p_{d}$, vapor pressure $p_{v}$, temperature $T$, and vapor mixing ratio $r_{v}$ for a parcel undergoing thermodynamical changes. In the appendix, we present a careful formulation of this equation that retains all the essential terms for this analysis.

Using in (8) the expression for the partial pressure of dry air for a mixture of moist air, (A4), that includes the effects of condensation, we have

$$
F_{z}=\rho_{d}\left(c_{p d}+r_{v}^{*} c_{p v}\right) \frac{\partial T}{\partial z}+\frac{\Lambda}{R_{d}} p_{d} \frac{\partial r_{v}^{*}}{\partial z}-g \rho+\left(F_{v d}+F_{d v}\right)
$$

Here, we made explicit in the parenthesis the cancellation of the internal force pair $F_{v d}+F_{d v}=0$. MGH proposes that the equation should be instead (e.g., Gorshkov et al. 2012)

$$
F_{z, \mathrm{MGH}}=\rho_{d}\left(c_{p d}+r_{v}^{*} c_{p v}\right) \frac{\partial T}{\partial z}+\frac{\Lambda}{R_{d}} p_{d} \frac{\partial r_{v}^{*}}{\partial z}-g \rho+F_{v d} .
$$

Here is again evident the flaw in MGH's theory by including $F_{v d}$ in the vertical motion equation while omitting $F_{d v}$ and not addressing the role of dry air in the mixture. Since these two forces cancel, their net effect on the moist atmosphere as a whole is zero. Thus, they have no effect on geophysical fluid dynamics. This is precisely the same argument that we presented from the mechanical approach, but now addressing explicitly that the mechanical picture holds even in the presence of condensation, consistent with the thermodynamical perspective.

\section{MGH and the atmospheric circulation}

MGH was originally proposed to improve the understanding of the airflow and moisture transport over forests. Nevertheless, MGH never made any serious efforts in presenting the existing theory (or theories) on the maintenance of flows in a moist atmosphere. For example, Carnot (1824) recognized that the generation of convective motion by clouds is similar to the production of mechanical work by a steam engine (Pauluis and Held 2002a), and later works like Brunt (1941) and Lorenz (1967) used the heat engine concept to explain Earth's general circulation (Rennó and Adams 2001). The atmosphere can be approached as a heat engine that produces mechanical work by transporting energy from warm to cold regions. This work is used primarily to sustain atmospheric motions and to lift water substance, and it is lost as a result of a turbulent energy cascade or in the wake of falling hydrometeors (Pauluis et al. 2000). The heat engine approach has been extensively applied to discuss various atmospheric circulations, such as moist convection, tornadoes, dust devils, and hurricanes (e.g., Bister and Emanuel 1998; Emanuel 2003, 1986; Emanuel and Bister 1996; Rennó et al. 1998; Rennó and Ingersoll 1996; Rennó and Adams 2001; Pauluis and Held 2002a; Adams and Rennó 2005; Pauluis 2011; Singh and O'Gorman 2016; Laliberté et al. 2015).

Hydrologic circulations, including those over forests, are readily explained using the Gibbs equation for a moist air parcel (Pauluis 2011; Raymond 2013) applied to such a circulation,

$$
\begin{aligned}
\frac{d p}{\rho} & =d h-T d s-\mu_{v} d r_{v}-\mu_{l} d r_{l} \\
& =d h-T d s-\mu_{v} d r_{T}-\left(\mu_{l}-\mu_{v}\right) d r_{l},
\end{aligned}
$$

where $T$ is the temperature, $d s$ is the change in the entropy of a parcel containing a unit mass of dry air plus changing mixing ratios of water vapor $d r_{v}$ and condensate $d r_{l}, r_{T}=r_{v}+r_{l}$ is the total cloud water mixing ratio, $d h$ is the change in enthalpy per unit mass of dry air, and $d p / \rho$ is the ratio of pressure change to parcel density. The quantities $\mu_{v}$ and $\mu_{l}$ are the chemical potentials of vapor and condensate (also equal to the respective specific Gibbs free energies). Note that this is a parcel theory, so that entrainment of environmental air is not considered. The ice phase of condensate is also ignored for simplicity.

Pauluis (2011) derived approximate expressions for $\mu_{v}$ and $\mu_{l}$ :

$$
\begin{aligned}
& \mu_{l}=\frac{C_{l}\left(T-T_{R}\right)^{2}}{2 T_{R}}, \\
& \mu_{v}=\mu_{l}+R_{v} T \ln H,
\end{aligned}
$$

where $C_{l}$ is the specific heat of liquid water, $R_{v}$ is the gas constant for water vapor, and $H$ is the relative humidity. 
Inside cloud air $H=1, \mu_{v}=\mu_{l}$, and the last term on the right-hand side of (12) vanishes.

Equation (12) along with the Kelvin circulation theorem gives the circulation tendency around a closed path,

$$
\frac{d \Gamma}{d t}=-\oint \frac{d p}{\rho}=\oint T d s+\oint\left(\mu_{v} d r_{v}+\mu_{l} d r_{l}\right),
$$

where the $d h$ term drops out because it is the integral around a closed loop of a perfect differential. Let us consider an idealized hydrological circulation that follows a parcel of air as shown in Fig. 1. Point 1 is moist air starting in the boundary layer. It ascends through the troposphere from 1 to 2 , raining out condensate as it forms, so that $r_{l}=0$ through the entire cycle. In this segment the entropy remains constant while the temperature decreases. In addition, the vapor mixing ratio decreases while the chemical potential of the vapor remains essentially fixed. [As with Pauluis (2011), we assume that $C_{l}=0$, which means that the relative humidity of the parcel controls the chemical potential. Pauluis (2011) also found that nonzero $C_{l}$ had little effect.]

In segment 2 to 3 , the parcel descends to the surface with constant mixing ratio and decreasing chemical potential and relative humidity. Descent is being driven by radiative cooling, so the entropy decreases as the temperature increases. Finally in segment 3 to 1 , the parcel moistens in the boundary layer at constant temperature and the entropy increases to its original value.

The values of the line integrals are equal to the areas of the respective triangles with a clockwise circuit being positive, yielding

$$
\frac{d \Gamma}{d t}=\frac{1}{2}\left[\left(S_{1}-S_{3}\right)\left(T_{1}-T_{2}\right)-\left(r_{v 1}-r_{v 3}\right)\left(\mu_{v 3}-\mu_{v 2}\right)\right] .
$$

The first term on the right-hand side of this equation tends to increase the circulation, while the second one tends to decrease it. This is an example of the "steam cycle" of Pauluis (2011). In this case, the first term is of order $L_{L} \Delta r$ and the second is of order $R_{v} T \ln (H) \Delta r$, where $L_{L}$ is the latent heat of condensation, $R_{v}$ is the gas constant for water vapor, and $\Delta r=r_{v 1}-r_{v 2}$. Typical numerical values indicate that the second term is an order of magnitude smaller than the first in this case, which means that the integral of $T d s$ dominates in (15).

This simple analysis is in agreement with the idea that the hydrologic cycle plays a central role in determining the amount of work that is generated and dissipated in
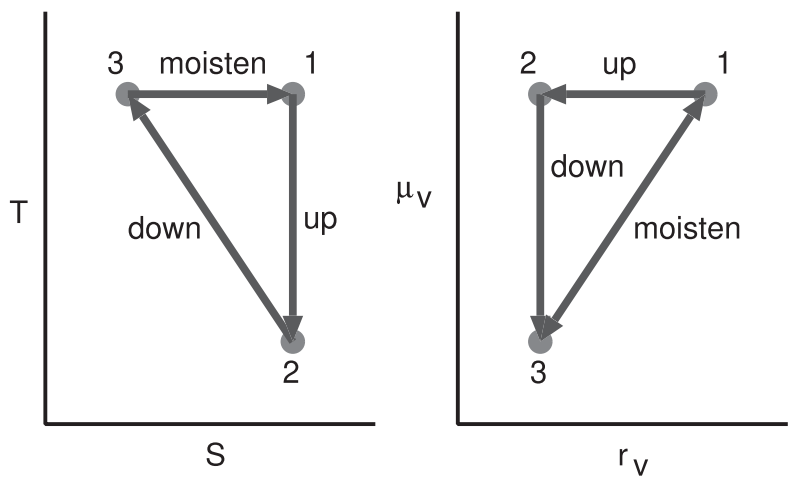

FIG. 1. Idealized hydrological circulation.

a moist atmosphere (Pauluis 2011). The hydrologic cycle is an important contributor to the generation of irreversible entropy, suggesting that moist processes, including the frictional dissipation associated with falling hydrometeors, tend to limit the work output of the atmospheric heat engine (Pauluis et al. 2000; Pauluis and Held 2002a,b; Pauluis 2011; Pauluis and Dias 2012; Laliberté et al. 2015). The work output is always less than that of an equivalent Carnot cycle, and it is constrained by the power necessary to maintain the hydrological cycle (Laliberté et al. 2015).

The arguments show the importance of surface evaporation to the maintenance of atmospheric circulations over forests and oceans. However, unlike in $\mathrm{MGH}$, the evaporation does not have to be collocated with the precipitation. Thus, for example, evaporation in the trade wind regions feeds the Hadley circulation, and the deep convection in its ascending branch does not depend solely on surface evaporation occurring directly beneath it.

\section{Conclusions}

We present a careful analysis of the physical principles behind the alleged new unstudied force, associated with the condensation of water vapor in the gravitational field of Earth, proposed by MGH. The hypothesis attracted the attention of the academic community by claiming that this force drives the atmospheric circulation. Also, researchers interested in a better understanding of the interaction among the forests, the hydrological cycle, and the atmospheric circulation were attracted. Clearly this proposal is in conflict with the standard theory, where the buoyancy force is a dominant driver of atmospheric motions. Despite its importance, doubts about the proposal remained, because there were not solid physical explanations of the flaws in MGH. In our opinion, this is 
no longer the case; we show definitively that the hypothesis is incorrect.

The primary conclusion of this paper is that the theory of MGH violates Newton's third law. This is because their "evaporative force" is actually an internal force between the dry-air and vapor components of the atmosphere. This force occurs because the actual scale height of water vapor in Earth's atmosphere is less than would occur if the water vapor by itself were in hydrostatic equilibrium independent of the dry air component. As a result, the negative upward gradient in the partial pressure of water vapor exceeds the downward gravitational force on the vapor. The water vapor does not expand rapidly upward as a result of this imbalance because of the downward force of the dryair component on the water vapor, as mediated by molecular collisions. Since this downward force is an internal force between the dry and vapor components of the air, there is a corresponding upward force exerted by the water vapor on the dry air. These equal and opposite forces constitute a Newton's third law pair. MGH's evaporative force is nothing more than the upward force of water vapor on the dry air. Their theory is invalid, because it fails to include the other component of the Newton's third law pair, namely, the downward force of the dry air on the water vapor. Since these two forces cancel, their net effect on the moist atmosphere as a whole is zero. Thus, they have no effect on geophysical fluid dynamics.

MGH was originally proposed to improve the understanding of the airflow and moisture transport over forests. Nevertheless, MGH never made any serious efforts in presenting the existing theory (or theories) on the maintenance of flows in a moist atmosphere. This is clear from MGH's difficulty in understanding the production of work in thermally direct circulation, by asserting that the ascending and descending components of work done by the buoyancy force cancel, resulting in a significant decrease in the energy released by atmospheric circulations. We showed using the Kelvin circulation theorem that the latent and sensible heating injected into the circulation at low levels by surface fluxes have a net positive effect on the circulation. The importance of evaporation to the maintenance of atmospheric circulations is thus clear, but it does not have to coincide with locations of strong precipitation. The explanation for the distribution of precipitation over regions such as the Amazon must be sought elsewhere, perhaps by studying the full water budget for such regions.

We agree with MGH that the water cycle plays an important role in the atmospheric circulation as we described. We think that it is worthwhile to pursue research to understand better the role of evaporation in the relationship between forests and climate.

Acknowledgments. Fellowships from the Universidad Nacional de Colombia and from the City of Medellin Sapiencia-Enlaza Mundos made the work of the first author possible. Jaramillo also thanks George Kiladis for useful discussions during his visit to the NOAA ESRL Physical Sciences Division (PSD) at Boulder, Colorado. Raymond was supported by U.S. National Science Foundation Grant 1546698. Mesa was supported by Universidad Nacional de Colombia. We thank Anastassia Makarieva for her useful comments on a previous version of this manuscript. We thank David Adams for his helpful comments and reference suggestions. We are grateful for the suggestions from one anonymous reviewer and from Olivier Pauluis, who disclosed his name as reviewer, offering us valuable comments and reference suggestions that helped to improve the paper.

\section{APPENDIX}

\section{The Pressure Gradient from the Moist Entropy Equation}

We must refer to the moist entropy equation to study the thermodynamic changes for a moist air parcel. This equation links the dry partial pressure $p_{d}$, vapor pressure $p_{v}$, temperature $T$, and vapor mixing ratio $r_{v}$ for a moist parcel undergoing thermodynamic changes including condensation. In this appendix, we approach this equation without using common simplifications in the literature, so that the contribution of water vapor is explicit. For saturated moist air, it takes the form (Raymond 2013)

$$
\begin{aligned}
\eta= & \left(c_{p d}+r_{v}^{*} c_{p v}\right) \ln \left(\frac{T}{T_{F}}\right)-R_{d} \ln \left(\frac{p_{d}}{p_{R}}\right) \\
& -r_{v}^{*} R_{v} \ln \left(\frac{p_{v}^{*}}{e_{\mathrm{SF}}}\right)+\frac{L_{L}\left(T_{F}\right) r_{v}^{*}}{T_{F}},
\end{aligned}
$$

where $\eta$ is the moist entropy per unit mass of dry air, $c_{p d}$ and $c_{p v}$ are the respective specific heats of dry air and vapor at constant pressure, and $r_{v}^{*}$ is the mixing ratio of saturated vapor. Other terms in (A1) are as follows: $R_{d}$ and $R_{v}$ are the gas constants for dry air and vapor, $L_{L}$ and $e_{\mathrm{SF}}$ are, respectively, the latent heat of condensation and the saturation vapor pressure at a reference temperature $T_{F}$ that is usually taken to be the freezingpoint temperature, and $p_{R}$ is a reference pressure usually taken to be $1000 \mathrm{hPa}$. For simplicity, we ignored the contribution of the liquid water to the specific entropy. 
Differentiating (A1) and assuming that the specific heats are relatively constant in the ranges of interest in Earth's atmosphere, we have

$$
\begin{aligned}
d \eta= & \left(c_{p d}+r_{v}^{*} c_{p v}\right) d \ln (T)-R_{d} d \ln \left(p_{d}\right) \\
& -r_{v}^{*} R_{v} d \ln \left(p_{v}^{*}\right)+\Lambda d r_{v}^{*},
\end{aligned}
$$

where $\Lambda$ collects all terms proportional to $d r_{v}^{*}$ and is given by

$$
\Lambda=\frac{L_{L}\left(T_{F}\right)}{T_{F}}+c_{p v} \ln \left(\frac{T}{T_{F}}\right)-R_{v} \ln \left(\frac{p_{v}^{*}}{e_{\mathrm{SF}}}\right) .
$$

Equation (A2) differs from the equation present in the literature in that it includes explicitly the term $r_{v}^{*} R_{v} d \ln \left(p_{v}^{*}\right)$, which is usually neglected because of $r_{v}^{*} \ll r_{d}$, and the term $c_{p v} \ln \left(T / T_{F}\right)-R_{v} \ln \left(p_{v}^{*} / e_{\mathrm{SF}}\right)$, which is also neglected. For an adiabatic process, that is, $d \eta=0$, from (A2) it is straightforward to show that in the steady state the vertical pressure gradient for dry air inside this column is given by

$$
\frac{\partial p_{d}}{\partial z}=\rho_{d}\left(c_{p d}+r_{v}^{*} c_{p v}\right) \frac{\partial T}{\partial z}+\frac{\Lambda}{R_{d}} p_{d} \frac{\partial r_{v}^{*}}{\partial z}-\frac{\partial p_{v}^{*}}{\partial z} .
$$

Notice that the last term in (A4) shows that the pressure gradient of dry air must adjust to the changes in the pressure gradient of water vapor due to the interactions of water vapor and dry air.

\section{REFERENCES}

Adams, D. K., and N. O. Rennó, 2005: Thermodynamic efficiencies of an idealized global climate model. Climate Dyn., 25, 801813, https://doi.org/10.1007/s00382-005-0071-y.

Bister, M., and K. A. Emanuel, 1998: Dissipative heating and hurricane intensity. Meteor. Atmos. Phys., 65, 233-240, https:// doi.org/10.1007/BF01030791.

Brunt, D., 1941: Physical and Dynamical Meteorology. Cambridge University Press, 428 pp.

Carnot, S., 1824: Réflexions sur la puissance motrice du feu et sur les machines propres à développer atte puissance (in French). Bachelier Libraire, 118 pp.

Emanuel, K. A., 1986: An air-sea interaction theory for tropical cyclones. Part I: Steady-state maintenance. J. Atmos. Sci., 43, 585-605, https:// doi.org/10.1175/1520-0469(1986)043<0585:AASITF>2.0.CO;2.

_, 2003: Tropical cyclones. Annu. Rev. Earth Planet. Sci., 31, 75104, https://doi.org/10.1146/annurev.earth.31.100901.141259.

_ , and M. Bister, 1996: Moist convective velocity and buoyancy scales. J. Atmos. Sci., 53, 3276-3285, https://doi.org/10.1175/ 1520-0469(1996)053<3276:MCVABS>2.0.CO;2.

Gorshkov, V. G., A. M. Makarieva, and A. V. Nefiodov, 2012: Condensation of water vapor in the gravitational field. J. Exp. Theor. Phys., 115, 723-728, https://doi.org/10.1134/S106377611209004X.

Holton, J. R., and G. J. Hakim, 2013: An Introduction to Dynamic Meteorology. 5th ed. Academic Press, 532 pp.

Lackmann, G. M., and R. M. Yablonsky, 2004: The importance of the precipitation mass sink in tropical cyclones and other heavily precipitating systems. J. Atmos. Sci., 61, 1674-1692, https:// doi.org/10.1175/1520-0469(2004)061<1674:TIOTPM>2.0.CO;2.

Laliberté, F., J. Zika, L. Mudryk, P. J. Kushner, J. Kjellsson, and K. Döös, 2015: Constrained work output of the moist atmospheric heat engine in a warming climate. Science, 347, 540543, https://doi.org/10.1126/science.1257103.

Lorenz, E., 1967: The Nature and Theory of the General Circulation of the Atmosphere. World Meteorological Organization, $161 \mathrm{pp}$.

Makarieva, A. M., and V. G. Gorshkov, 2007: Biotic pump of atmospheric moisture as driver of the hydrological cycle on land. Hydrol. Earth Syst. Sci., 11, 1013-1033, https://doi.org/10.5194/ hess-11-1013-2007.

_, and —-, 2009a: Condensation-induced dynamic gas fluxes in a mixture of condensable and non-condensable gases. Phys. Lett., 373A, 2801-2804, https://doi.org/10.1016/ j.physleta.2009.05.057.

and - , 2009b: Condensation-induced kinematics and dynamics of cyclones, hurricanes and tornadoes. Phys. Lett., 373A, 4201-4205, https://doi.org/10.1016/j.physleta.2009.09.023.

$\longrightarrow$, and -2009 c: Reply to A. G. C. A. Meesters et al.'s comment on "Biotic pump of atmospheric moisture as driver of the hydrological cycle on land." Hydrol. Earth Syst. Sci., 13, 1307-1311, https://doi.org/10.5194/hess-13-1307-2009.

—, and _ 2010: The biotic pump: Condensation, atmospheric dynamics and climate. Int. J. Water, 5, 365-385, https://doi.org/ 10.1504/IJW.2010.038729.

, — - D. Sheil, A. D. Nobre, and B.-L. Li, 2013: Where do winds come from? A new theory on how water vapor condensation influences atmospheric pressure and dynamics. Atmos. Chem. Phys., 13, 1039-1056, https://doi.org/10.5194/ acp-13-1039-2013.

, — , and A. V. Nefiodov, 2014: Condensational power of air circulation in the presence of a horizontal temperature gradient. Phys. Lett., 378A, 294-298, https://doi.org/10.1016/ j.physleta.2013.11.019.

,-- , and -2015 : Empirical evidence for the condensational theory of hurricanes. Phys. Lett., 379A, 2396-2398, https://doi.org/10.1016/j.physleta.2015.07.042.

,,-- A. V. Chikunov, D. Sheil, A. D. Nobre, and B.- L. Li, 2017: Fuel for cyclones: The water vapor budget of a hurricane as dependent on its movement. Atmos. Res., 193, 216-230, https://doi.org/10.1016/j.atmosres.2017.04.006.

Meesters, A. G. C. A., A. J. Dolman, and L. A. Bruijnzeel, 2009: Comment on "Biotic pump of atmospheric moisture as driver of the hydrological cycle on land" by A. M. Makarieva and V. G. Gorshkov, Hydrol. Earth Syst. Sci., 11, 1013-1033, 2007. Hydrol. Earth Syst. Sci., 13, 1299-1305, https://doi.org/ 10.5194/hess-13-1299-2009.

Pauluis, O., 2011: Water vapor and mechanical work: A comparison of Carnot and steam cycles. J. Atmos. Sci., 68, 91-102, https://doi.org/10.1175/2010JAS3530.1.

— , and I. M. Held, 2002a: Entropy budget of an atmosphere in radiative-convective equilibrium. Part I: Maximum work and frictional dissipation. J. Atmos. Sci., 59, 125-139, https://doi.org/ 10.1175/1520-0469(2002)059<0125:EBOAAI > 2.0.CO;2.

$\longrightarrow$, and — 2002b: Entropy budget of an atmosphere in radiative-convective equilibrium. Part II: Latent heat transport and moist processes. J. Atmos. Sci., 59, 140-149, https:// doi.org/10.1175/1520-0469(2002)059<0140:EBOAAI >2.0.CO;2. , and J. Dias, 2012: Satellite estimates of precipitation-induced dissipation in the atmosphere. Science, 335, 953-956, https:// doi.org/10.1126/science.1215869. 
V. Balaji, and I. M. Held, 2000: Frictional dissipation in a precipitating atmosphere. J. Atmos. Sci., 57, 989-994, https://doi.org/10.1175/1520-0469(2000)057<0989: FDIAPA $>2.0 . \mathrm{CO} ; 2$.

Raymond, D. J., 2013: Sources and sinks of entropy in the atmosphere. J. Adv. Model. Earth Syst., 5, 755-763, https://doi.org/ 10.1002/jame.20050.

Rennó, N. O., and A. P. Ingersoll, 1996: Natural convection as a heat engine: A theory for CAPE. J. Atmos. Sci., 53, 572-585, https:// doi.org/10.1175/1520-0469(1996)053<0572:NCAAHE >2.0.CO;2; Corrigendum, 53, 1355, https://doi.org/10.1175/1520-0469(1996) 053<1355: $>2.0 . \mathrm{CO} ; 2$.
- , and D. K. Adams, 2001: The convective heat engine. Recent Research Developments in Atmospheric Science, S. Pandalai, Ed., Research Signpost, 1-14.

— M. L. Burkett, and M. P. Larkin, 1998: A simple thermodynamical theory for dust devils. J. Atmos. Sci., 55, 3244-3252, https:// doi.org/10.1175/1520-0469(1998)055<3244:ASTTFD>2.0.CO;2.

Singh, M. S., and P. A. O'Gorman, 2016: Scaling of the entropy budget with surface temperature in radiative-convective equilibrium. J. Adv. Model. Earth Syst., 8, 1132-1150, https:// doi.org/10.1002/2016MS000673.

Wallace, J., and P. Hobbs, 2006: Atmospheric Science: An Introductory Survey. Academic Press, 504 pp. 